\title{
New challenges in the application of biocompatible silver nanoparticles in enology: Antimicrobial capacity, digestibility and potential cytotoxicity
}

\author{
I. Gil-Sánchez ${ }^{1}$, M. Monge ${ }^{2}$, A. Bernáldez ${ }^{1}$, A. Tamargo ${ }^{1}$, C. Cueva ${ }^{1}$, D. González de Llano ${ }^{1}$, B. Bartolomé1, \\ and M.V. Moreno-Arribas ${ }^{1, a}$ \\ ${ }^{1}$ Instituto de Investigación en Ciencias de la Alimentación (CIAL), CSIC-UAM, c/ Nicolás Cabrera 9, 28049 Madrid, Spain \\ ${ }^{2}$ Universidad de La Rioja, Dpto. de Química, c/ Madre de Dios 51, 26004, Logroño, La Rioja, Spain
}

\begin{abstract}
In the search for new substitutes to the use of sulfur dioxide $\left(\mathrm{SO}_{2}\right)$ in oenology, a new line of research based on nanotechnology has been launched. Within this line, this paper evaluates the suitability of two new biocompatible silver nanoparticles (Ag-NPs) to control microbial growth in wine as well as their stability and potential risks at intestinal level. Antimicrobial activities against spoilage wine microorganisms were carried out in both culture media and wines. In addition, in vitro simulations (static and dynamic) were conducted to monitor the passage of Ag-NPs through the buco-gastrointestinal tract and also to assess their effects on the gut microbiota and intestinal epithelial cells. Both Ag-NPs proved to be highly efficient for the control of wine undesirable bacteria in culture media and microvinification assays, and to a lesser extent, for reducing Brettanomyces populations in wine. Furthermore, undigested Ag-NPs as their corresponding digests after gastrointestinal simulation were not toxic to intestinal microbiota either epithelial cells, at least at the conditions used in these experiments.
\end{abstract}

\section{Introduction}

Sulfur dioxide $\left(\mathrm{SO}_{2}\right)$ is one of the most efficient additives used in winemaking due to its two important roles. Firstly, $\mathrm{SO}_{2}$ is able to selectively inhibit the growth of wine undesirable microorganisms, mainly lactic acid bacteria (LAB), and, secondly, it acts as an antioxidant, safeguarding the integrity of the wine and protecting it against browning.

However, several studies have reported that high exposures to this additive can produce organoleptic alterations in the final product and can cause health adverse effects in sensitive people [1]. Because of this, the sulfating practice is actually strictly controlled in musts and wines.

A general concern for the development of new alternatives that can replace or complement the action of $\mathrm{SO}_{2}$ has been currently promoted. Recent proposed alternatives include the addition of "natural antimicrobial agents" such as bacteriocins, lysozyme and polyphenols as well as the application of physical methods, such as pulsed electric field, ultrasound, ultraviolet radiation, low electric current and high pressure [2,3]. Another potential alternative to the use of $\mathrm{SO}_{2}$ are silver nanoparticles (Ag-NPs) [3,4].

The antimicrobial effects of silver have been known for years, however, it is now when its use has gained prominence in the agro food industry [4]. Some recent papers have evaluated the effects of antiseptic

$\overline{\text { a e-mail: victoria.moreno@csic.es }}$ kaolin-silver complex for substituting the use of sulfites in winemaking $[5,6]$.

In the case of the silver nanoparticles, these biomaterials are much more active and soluble than a similar bulk amount of silver. Nevertheless, so far, studies on the use silver nanoparticles in the field of oenology as antimicrobial agents are very scarce, and, there is no information available on the effect of these nanomaterials on healthy human [3]. Thus, the aim of the present study was to assess the ability of two new biocompatible silver nanoparticles to control the microbial growth in wines as well as to evaluate the potential digestive risks related to their intake.

\section{Materials and methods}

\subsection{Silver nanoparticles}

The two biocompatible silver nanoparticles (coating by polyethylenglycol and glutathione) were synthetized following the methodology described by García-Ruíz et al. [3].

The polyethylene glycol-stabilized silver nanoparticles identified as PEG-Ag NPs had a final content of silver of $20 \%$ and an average size between $5-25 \mathrm{~nm}$, whereas the glutathione-stabilized silver nanoparticles designated as GSH-Ag NPs had a final concentration of silver of $0.197 \mathrm{mg} / \mathrm{mL}$ and an average size between $10-50 \mathrm{~nm}$.

Both Ag-NPs were characterized by transmission electron microscopy (TEM) and inductively coupled plasma (Fig. 1). 
A)

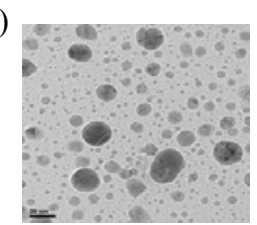

B)

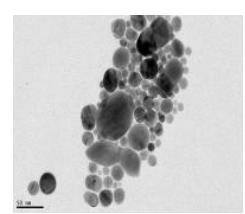

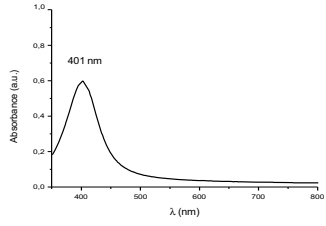

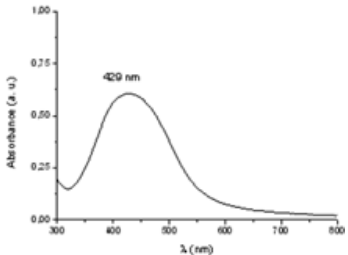

Figure 1. Transmission Electron Microscopy images of PEG- Ag NPs (A) and GLU-Ag NPs (B).

\subsection{Bacteria, culture media and antibacterial activity assay}

The antibacterial assays were determined by microtiter dilution method [3].

Eleven lactic acid bacteria (LAB) strains belonging to $P$. pentosaceus $(\mathrm{n}=4)$, L. casei $(\mathrm{n}=3)$, L. plantarum $(\mathrm{n}=1)$, O. oeni $(\mathrm{n}=3)$ (Table 1$)$ were selected from the bacterial culture collection of CIAL (Instituto de Investigación en Ciencias de la Alimentación, CSICUAM). In addition, two acetic acid bacteria (AAB) strains belonging to Spanish Type Culture Collection (CECT), A. aceti CECT-298 and G. oxydans CECT360 were also selected for this study. Lactobacillus, Pediococcus and $\mathrm{O}$. oeni strains were grown at $30^{\circ} \mathrm{C}$ in MRS medium. $\mathrm{AAB}$ were cultivated at $30^{\circ} \mathrm{C}$ in $\mathrm{GY}$ medium.

The antimicrobial activity of silver nanoparticles was evaluated as inhibition of bacterial growth during the stationary phase, and was expressed as the $\mathrm{IC}_{50}$ value (concentration of silver required to reduce $50 \%$ of cell growth). $\mathrm{IC}_{50}$ was determined using a non-linear regression model based on the equation for a sigmoid dose-response curve $(\mathrm{R} 2>0.9)$ (GraphPadPrism v.4.03, GraphPad, La Jolla, USA).

\subsection{Antibacterial activity assay in microvinification conditions}

The wine used in this study was a young white wine (var. Airén, vintage 2014), kindly provided by a winery (La Mancha Designation of Origin). This wine was selected because of its low $\mathrm{SO}_{2}$ content (free $\mathrm{SO}_{2} \leq 1 \mathrm{mg} / \mathrm{L}$ ).

The wine was divided into four glass flasks $(50 \mathrm{~mL})$, which were subjected to different treatments. These treatments consisted in adding: (a) PEG-Ag, (b) GSH$\mathrm{Ag}$, and (c) $\mathrm{SO}_{2}$ and (d) none antimicrobial agent (control wine). The tested silver nanoparticles concentrations were calculated from $\mathrm{IC}_{50}$ of $L$. plantarum (Table 1), whereas, in the case of $\mathrm{SO}_{2}$ was used at the maximum dosage authorized $(160 \mathrm{mg} / \mathrm{L})$. All treated wines were incubated at $25^{\circ} \mathrm{C}$ for 30 days. Samples were collected at $0,1,3,7,14$, 21 and 30 days of incubation to perform bacterial counts (LAB and $\mathrm{AAB}$ ).

\subsection{Determination of antimicrobial activity of silver nanoparticles against Brettanomyces in wines}

A red wine was contaminated with Brettanomyces bruxellensis using a strain previously isolated from a spoiled wine. Wine, previously filtered $(20 \mu \mathrm{m})$, was inoculated with $B$. bruxellensis $\left(5-20 \times 10^{6} \mathrm{UFC} / \mathrm{mL}\right)$ and distributed in bottles of $125 \mathrm{~mL}$. Each bottle was subjected to different treatments that consisted in adding to the wine: (a) PEG-Ag NPs, (b) GSH-Ag NPs, (c) chitosan, (d) $\mathrm{SO}_{2}$ and (e) none antimicrobial agent (control wine). The concentration of chitosan used in this experiment, was $10 \mathrm{~g} / \mathrm{hL}$. To the rest of antimicrobial agents were used the same concentrations mentioned in above experiment.

Wine samples were collected at 15 and 30 days to perform Brettanomyces counting in BrettAllert medium (Agrovin S.A, Ciudad Real, Spain). Analyses were done in duplicate.

\subsection{In vitro buco-gastrointestinal static simulation of silver nanoparticles and cytotoxicity assays}

The oral-gastrointestinal simulations of biocompatible silver nanoparticles were conducted following the harmonized static digestion method developed within the COST Action INFOGEST [7]. This method is comprised to three stages that mimic the oral, gastric and small intestinal phases of digestion in vivo, by addition of synthetic fluids (salivary fluid, gastric fluid and intestinal fluid).

Serial dilutions of undigested and digested silver nanoparticles [from $0.44 \mathrm{mg} / \mathrm{mL}$ to $688 \mu \mathrm{g} / \mathrm{mL}$ for PEGAg NPs, and from $0.309 \mathrm{~mL} / \mathrm{mL}$ to $483 \mu \mathrm{L} / \mathrm{mL}$ for GSHAg NPs] were used for cytotoxicity testing on proliferative and differentiated Caco-2 cells, using the colorimetric 3-(4,5-dimethylthiazol-2-yl)-2,5- diphenyltetrazolium bromide (MTT) assay [8]. The Caco-2 cells were obtained from American Type Culture Collection (ATCC). The results were expressed as percentage of cell viability relative to control. The optical density of control was established as the maximum viability (100\%).

\subsection{In vitro gastrointestinal dynamic simulation of silver nanoparticles and effect on gut microbiota}

With the aim to investigate the possible effects of silver nanoparticles on gut microbiota, a dynamic gastrointestinal tract model, registered as simgi ${ }^{\circledR}$, was used. The simgi ${ }^{\circledR}$ is a multi-compartmental dynamic model controlled by a central computer, which led simulate jointly the conditions of the stomach, small intestine and three colon regions (ascending, transverse and descending colon) $[9,10]$. In addition, this model allows developing and maintaining a stable and colon specific microbial ecosystem.

Two independent assays were carried out using fecal samples from the two healthy volunteers. The operation of the model was optimized previously [10]. Briefly, the simgi ${ }^{\circledR}$ was inoculated with faecal solution and, next, and after a 2-week stabilization period the system was fed with a single dose of GSH-Ag NPs. The system was monitored during one week after which it was considered that the system returned to initial conditions. 
Table 1. $\mathrm{IC}_{50}$ data of silver nanoparticles and potassium metabisulphite against the bacteria studied (adapted from GarcíaRuíz et al., 2015 [3]).

\begin{tabular}{|c|c|c|c|}
\hline & \multicolumn{3}{|c|}{$\mathrm{IC}_{50}(\mu \mathrm{g} \mathrm{Ag} / \mathrm{mL})$} \\
\hline & $\begin{array}{c}\text { PEG-Ag } \\
\text { NPs }\end{array}$ & GSH-Ag & $\mathbf{K} \mathbf{2}_{5}$ \\
\hline \multicolumn{4}{|l|}{ LAB } \\
\hline P. pentosaceus CIAL-16 & 120 & 62 & 590 \\
\hline P. pentosaceus CIAL-49 & 86 & 70 & 610 \\
\hline P. pentosaceus CIAL-85 & 470 & 50 & 550 \\
\hline P. pentosaceus CIAL-86 & 410 & 69 & 600 \\
\hline L. casei CIAL-51 & 232 & 65 & 540 \\
\hline L. casei CIAL-52 & 380 & 46 & 310 \\
\hline L. casei CIAL-92 & 370 & 17 & 820 \\
\hline L. plantarum CIAL-121 & 88 & 61 & 540 \\
\hline O. oeni CIAL-117 & 208 & 1.7 & 360 \\
\hline O. oeni CIAL-118 & 202 & 1.2 & 440 \\
\hline O. oeni CIAL-119 & 210 & 2.4 & 560 \\
\hline \multicolumn{4}{|l|}{$\mathbf{A A B}$} \\
\hline A. aceti CECT-298 & 30 & 29 & 480 \\
\hline G. oxydans CECT-360 & 32 & 30 & 470 \\
\hline
\end{tabular}

During the course of the experiments, samples from the stomach, large intestine and the three colon compartments were collected before (day 0) and after of GSH-Ag NPs feeding (days 2, 3, 4, 5, 8 and 10), to perform further analyses.

To assess changes in gut microbiota after the GSH-Ag NPs feeding, simgi ${ }^{\circledR}$ samples from the colon compartments were decimal-diluted and plated on different types on selective media [Trypticase Soy Agar (BD) fortotalaerobes; Wilkins Chalgrenagar (BD) fortotalanaerobes; MacConkeyagar (BD) for Enterobacteriaceae spp.; Enterococcus agar (BD) for Enterococcus spp.; MR Sagar (Pronadisa) for lactic acid bacteria and Manitol (BD) for S.aureus spp]. All plates were incubated at $37^{\circ} \mathrm{C}$ for $48 \mathrm{~h}$ in an anaerobic cabinet (BACTRON Anaerobic/Environmental Chamber, SHELLAB), except Manitol and TSA that were incubated in aerobic conditions.

\section{Results and discussion}

\subsection{Antimicrobial activity of PEG-Ag NPs and GSH-Ag NPs in culture media}

As shown in Table 1, both silver nanoparticles were able to inhibit the growth of the main wine $\mathrm{LAB}$ and $\mathrm{AAB}$ in culture media. PEG-Ag NPs resulted in being more effective (lower $\mathrm{IC}_{50}$ values) against Gram-negative strains (AAB) than against Gram-positive strains (LAB), whereas glutathione-stabilized GSH-Ag NPs resulted in being extraordinarily effective against $O$. oeni strains ( $\mathrm{IC}_{50} \sim 1 \mu \mathrm{g} / \mathrm{mL}$, Ag concentration) [3].

It is important to remark that, in general, the tested bacterial populations were more sensitive to Ag-NPs action than $\mathrm{K}_{2} \mathrm{~S}_{2} \mathrm{O}_{5}$, thus confirming their antimicrobial effects in wine microbiota.

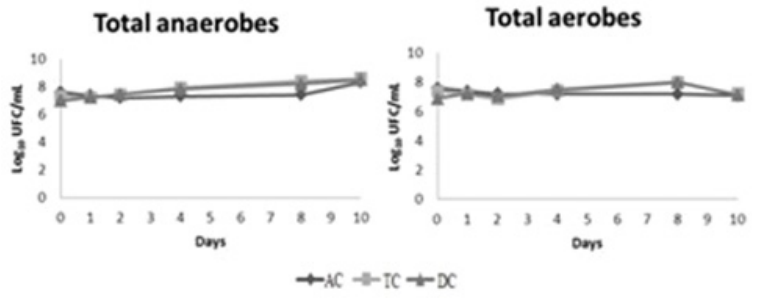

Figure 2. Total anaerobes and aerobes bacteria counts performed in ascending colon (AC), transverse colon (TC), and descending colon (DC), before (day 0) and after of feeding of simgi ${ }^{\mathrm{B}}$ with silver nanoparticles (days from 1 up to 10) for volunteer \#1.

\subsection{Evaluation of antimicrobial activity of silver nanoparticles in microvinification trials}

Results from the microvinification trials showed that wines treated with both Ag-NPs completely inhibited the LAB and $\mathrm{AAB}$ populations, while that, control wine practically maintained the initial bacterial counts (data not shown).

Besides, it is important mention that after the first 24 hours in contact with wine, the PEG-Ag NPs were able to almost completely reduced $\mathrm{LAB}$ and $\mathrm{AAB}$ populations, providing an effect slight higher than the $\mathrm{SO}_{2}$.

\subsection{Evaluation of the susceptibility of Brettanomyces to silver nanoparticles in wines}

Silver nanoparticles, unlike $\mathrm{SO}_{2}$ and chitosan, were not able to completely remove Brettanomyces populations in wines. However, it is worth highlighting that, after 30 days of treatment, both PEG-Ag and GSH-Ag NPs decreased the Brettanomyces counts almost two log units, respect to wine control (data not shown).

\subsection{Evaluation of cytotoxicity of silver nanoparticles on Caco-2 cells}

After demonstrating the effectiveness of both Ag-NPs as antimicrobial agents in wines, the next step was to assess the safety of these nanomaterials for humans, in particular, on intestinal epithelium.

The results showed that none of the undigested AgNPs proved toxic to Caco-2 cells, at microbiologically active concentrations. In addition, these results were confirmed with the data obtained after oral-gastrointestinal simulation.

Previous studies also have discarded cytotoxicity effects on the Caco-2 cells by undigested silver nanoparticles [11].

\subsection{Effect of GSH-Ag NPs on gut microbiota using a dynamic gastrointestinal model}

With the purpose of deepening our understanding of the safety of silver nanoparticles at digestive level, the effects of the Ag-NPs stabilized with glutathione on gut microbiota were assessed using a dynamic gastrointestinal model.

In general, the counts of major microbial groups remained stable, over whole experiments, detecting changes $\log 10 \mathrm{UFC} \leq 1$. As an example, Figure 2 shows the counts of total anaerobes and aerobes bacteria after feeding with silver nanoparticles for volunteer \#1. 
These results demonstrated that GSH-Ag NPs, at the concentration tested, produced no significant changes in the viability of microbiota present in the gastrointestinal tract.

In conclusion, these finding confirmed the potential of silver nanoparticles to control microbial processes in winemaking. They also seemed to discard toxicological effects of these nanoparticles on gut microbiota and intestinal epithelial cells in vitro, at least at the conditions tested. Nevertheless, further studies are required to ensure food security of these biomaterials in vivo.

This work was funded by the Spanish Ministry of Economy and Competitiveness (PRI-PIBAR-2011-1358 and AGL201564522-C2-R Projects), Comunidad de Madrid (ALIBIRD-CM S2013/ABI-2728), the Spanish Ministry of Education, Culture and Sport (grant FPU14/05760) and the 'Garantía Juvenil' Program (MINECO, CSIC).

\section{References}

[1] H. Vally, N.L. Misso, V. Madan. Clin. Exp. Allergy. 11, 1643-1651 (2009)

[2] A. García-Ruiz, B. Bartolomé, A.J. Martínez- Rodríguez, E. Pueyo, P.J. Martín-Álvarez, M.V. Moreno-Arribas. Food Control. 19, 835-841 (2008)

[3] A. García-Ruiz, J. Crespo, J.M. López-de-Luzuriaga, M.E. Olmos, M. Monge, M.P. Rodríguez-Álfaro,
P.J. Martín-Álvarez, B. Bartolome, M.V. Moreno-Arribas. Food Control. 50, 613-619 (2015)

[4] M. Monge, M.V. Moreno-Arribas. Wine safety, consumer preference, and human health. 51-69 (2016)

[5] P.M. Izquierdo-Cañas, E. García-Romero, B. Huertas-Nebreda, S. Gómez-Alonso. Food Control. 23, 73-81 (2012)

[6] T. Garde-Cerdán, P. López, R., Garijo, L. GonzálezArenzana, A.R. Gutiérrez, I. López- Alfaro, P. Santamaría. Aust. J. Grape Wine. 20, 51-61 (2003)

[7] M. Minekus, M. Alminger, P. Alvito, W. Weitschies, A. Brodkorb Food and Function. 5, 1113-1124 (2014)

[8] T. Mosmann. J. Immunol. Methods. 65, 55-63 (1983)

[9] E. Barroso, C. Cueva, C. Peláez, M.C. MartínezCuesta, T. Requena LWT Food Sci. Technol. 61, 283-289 (2015)

[10] C. Cueva, A. Jiménez-Girón, I. Muñoz- González, A. Esteban-Fernández, I. Gil- Sánchez, M. Dueñas, P.J. Martín-Álvarez, M.A. Pozo-Bayón, B. Bartolomé, M.V. Moreno- Arribas Food Res. Int. 72, 149-159 (2015)

[11] L. Böhmert, M. Girod, U. Hansen, R. Maul, P. Knappe, B. Niemann, S.M. Weidner, A.F. Thünemann, A. Lampen. Nanotoxicology. 8, 631-642 (2014) 